\title{
Capillary Surfaces in Negative Gravitational Fields
}

\section{Gerhard Huisken}

Institut für Angewandte Mathematik, Ruprecht-Karls-Universität, Im Neuenheimer Feld 294, D-6900 Heidelberg, Federal Republic of Germany

\section{Introduction}

We consider the equilibrium surface of a liquid of fixed volume in an upside down capillary tube. Let $\Omega \subset \mathbb{R}^{n}, n \geqq 2$ be the cross-section of the tube and assume that the top of the tube and the equilibrium surface can be represented as graphs of functions $\psi$ and $u$ on $\Omega$. Then the physical principle of virtual work leads to the consideration of the energy functional

$$
E(v)=\int_{\Omega}\left(1+|D v|^{2}\right)^{1 / 2} d x-\frac{\kappa}{2} \int_{\Omega} v^{2} d x+\int_{\hat{\delta} \Omega} \beta v d H_{n-1}
$$

where $\kappa$ (nonnegative) is the capillarity constant and $\beta \in L^{\infty}(\partial \Omega)$ is the cosine of the contact angle between the surface and the cylinder walls. We made the physical assumption that there is no contribution to the energy from the top of the tube, i.e. that the liquid 'wets' the obstacle $\psi$. For convenience of notation we reverse the coordinate system such that $\psi$ becomes the bottom of the tube and the gravitational field is upwards directed.

Because of the bad term

$$
-\frac{\kappa}{2} \int_{\Omega} v^{2} d x
$$

we can't expect any minimum of $E$ in

$$
K:=H^{1, \infty}(\Omega) \cap\{v \geqq \psi\} \cap\left\{\int_{\Omega} v-\psi d x=V\right\}
$$

where $V>0$ is the prescribed volume.

But we want to show here, that the corresponding variational inequality has a global regular solution, if we assume that at least one of the quantities $\kappa$ or $V$ is small enough.

Let $A$ be the minimal surface operator

$$
A=-D_{i}\left(a^{i}(p)\right)^{1}, \quad a^{i}=p_{i} \cdot\left(1+|p|^{2}\right)^{-1 / 2}
$$

1 Here and in the following we sum over repeated indices 
and let the functions

$$
\beta \in C^{1, \alpha}(\partial \Omega), \quad H=H(x, t) \in C^{1, \alpha}\left(\mathbb{R}^{n} \times \mathbb{R}\right)
$$

satisfy the conditions

and

$$
|\beta| \leqq 1-a ; \quad a>0
$$

$$
\sup _{\Omega} H(x, t) \leqq b \cdot(1+t) \quad t>0
$$

where $b$ is some positive constant and $H$ is introduced for greater generality. Then we can prove the following main theorem:

Theorem 0.1. Let $\Omega$ be a bounded domain of $\mathbb{R}^{n}, n \geqq 2$, with boundary of class $C^{2, \alpha}$, and let the functions $\beta$ and $H$ satisfy the conditions $(0.5)-(0.8)$. Then the variational inequality

where

$$
\langle A u+H(x, u)-\kappa u, v-u\rangle \geqq 0 \quad \text { for all } v \in K
$$

$$
\langle A u, \eta\rangle=\int_{\Omega} a^{i} D_{i} \eta d x+\int_{\partial \Omega} \beta \eta d H_{n-1}
$$

has a solution $u \in H^{1, \infty}(\Omega) \cap H^{2,2}(\Omega) \cap H_{\mathrm{loc}}^{2, \infty}(\Omega)$, if we assume $\psi \in C^{2}(\bar{\Omega})$ and if $\kappa$ or $V$ is small enough. The solution has continuous tangential derivatives at the boundary and in the case $n=2$ we have $u \in C^{1}(\bar{\Omega})$.

If we impose on $\psi$ the further assumption

$$
-a^{i}(D \psi) \cdot \gamma_{i} \geqq \beta \quad \text { on } \quad \partial \Omega
$$

where $\gamma$ is the outer unit normal to $\partial \Omega$, and if $\partial \Omega \in C^{3, \alpha}, \beta \in C^{1,1}(\partial \Omega)$ we have $u \in H^{2, \infty}(\Omega)$.

The proof is essentially based on a special a priori bound for the gradient of solutions to the problem

$$
\begin{aligned}
A u+H(x, u)=0 & \text { in } \Omega \\
-a^{i}(D u) \cdot \gamma_{i}=\beta & \text { on } \partial \Omega .
\end{aligned}
$$

Using ideas of Ural'ceva [18] and Gerhardt [4], we can show that this bound does not explicitly depend on $|H(\cdot, u(\cdot))|_{\Omega}$. In the second part of this article we shall look for a solution to the boundary value problem

$$
\begin{aligned}
A u-\kappa u+\lambda=0 & \text { in } \Omega \\
-a^{i} \cdot \gamma_{i}=\beta & \text { on } \partial \Omega
\end{aligned}
$$

where $\lambda$ is some parameter and $\kappa \geqq 0$ is small. It turns out that $(0.13)$ has always a solution for small $\kappa$, provided there is a solution in the case $\kappa=0$.

The article ends with a corresponding result for Dirichlet boundary conditions. 
The problem of a 'hanging drop' has been considered before by several authors, see $[2,11,12,14]$.

At this place the author wishes to thank Professor Gerhardt for having acquainted him with this problem and for helpful discussions.

Notations. We shall denote by $|\cdot|_{\Omega}$ the supremum norm on $\Omega$ and by $\|\cdot\|_{p}$ the norms of the $L^{p}$-spaces.

By $c=c(\ldots)$ we shall denote various constants whereas indices will be used, if a constant recurs at another place.

\section{Existence}

For technical reasons we assume

$$
\frac{\partial H}{\partial t} \geqq \tau>0
$$

and let $\tau$ tend to zero at the end of the proof. By considering the sideconditions as isoperimetric, we are led from (0.9) to the following approximating problems

$$
\begin{aligned}
A u+H(x, u)-\kappa u+\lambda+\mu \Theta_{\varepsilon}(u-\psi)=0 & \text { in } \Omega \\
-a^{i}(D u) \cdot \gamma_{i}=\beta & \text { on } \partial \Omega
\end{aligned}
$$

where $\lambda \in \mathbb{R}, 0<\mu \in \mathbb{R}$ are Lagrange multipliers and $\Theta_{\varepsilon}$ is a sequence of smooth monotone graphs tending to the maximal monotone graph $\Theta$ :

$$
\Theta(t)=\left\{\begin{array}{ll}
0, & t>0 \\
{[-1,0],} & t=0 \\
-1, & t<0
\end{array} \quad \Theta_{\varepsilon}(t)= \begin{cases}0, & t \geqq 0 \\
-1, & t \leqq-\varepsilon .\end{cases}\right.
$$

We want to obtain a solution to (1.2) by a fixed point argument and so we consider the related problem

$$
\begin{aligned}
A u+H(x, u)-\kappa \Phi+\lambda+\mu \Theta_{\varepsilon}(u-\psi) & =0 & & \text { in } \Omega \\
-a^{i}(D u) \cdot \gamma_{i} & =\beta & & \text { on } \partial \Omega
\end{aligned}
$$

for any $\Phi \in C^{1, \alpha}(\bar{\Omega})$.

From the results of Gerhardt $[4,5]$ we know

Lemma 1.1. For all $\Phi \in C^{1, \alpha}(\bar{\Omega})$ and $V>0$ there exists $\lambda(\Phi)$ and $u_{\Phi} \in C^{2}(\bar{\Omega})$, such that $u_{\Phi}$ solves the problem (1.4) and

$$
\int_{\Omega} u_{\Phi}-\psi d x=V
$$

The solution $u_{\Phi}$ and the Lagrange multiplier $\lambda(\Phi)$ are uniquely determined by $\Phi$ and $V$. 
In view of the Lemma there is an operator

$$
\begin{aligned}
T: C^{1, \alpha}(\bar{\Omega}) & \rightarrow C^{1, \alpha}(\bar{\Omega}) \\
\Phi & \rightarrow u_{\Phi} .
\end{aligned}
$$

It is our claim to show that $T$ has a fixed point, which would be a solution to (1.2). Therefore we want to use the following Lemma ([8], Corollary 10.2):

Lemma 1.2. Let $S$ be a closed convex set in a Banachspace $B$ and let $T$ be $a$ continuous mapping of $S$ into itself such that the image $T(S)$ is precompact. Then $T$ has a fixed point.

In order to verify the hypotheses of the Lemma we state the following a priori estimates, which will be proved in Sect. 2 and 3.

Theorem 1.1. Let $u$ be a solution to (1.4), which fulfills (1.5). Then we can take $\mu$ as large that

$$
u-\psi \geqq-3 \varepsilon
$$

where $\mu$ depends on $\varepsilon$ and tends to infinity when $\varepsilon$ tends to zero. Furthermore we have the upper bound

$$
u-\psi \leqq c \cdot\left(c+\kappa \cdot|\Phi|_{\Omega}\right) \cdot(V+\varepsilon)^{1 / n+1}
$$

the constants depending on $H, \psi, n, a, \Omega$ and $\partial \Omega$ but not on $\lambda, \mu, \varepsilon$ and $\tau$.

Remark, that as a consequence of (1.7) and (1.8), the term $|u-\psi|_{\Omega}$ tends to zero provided that $\mu$ tends to infinity and $\varepsilon$ and $V$ tend to zero.

Theorem 1.2. A solution of (1.4) satisfies in the whole domain $\Omega$

$$
\log |D u| \leqq c+c \cdot\left(\kappa \cdot|D \Phi|_{\Omega} \cdot|u-\psi|_{\Omega}\right)
$$

where the constants depend on known quantities but not on $\lambda, \mu, \varepsilon$ and $\tau$.

Now let

$$
S_{M}:=\left\{\left.\Phi \in C^{1, \alpha}(\bar{\Omega})|| \Phi\right|_{C^{1}} \leqq M\right\} .
$$

From the two theorems we deduce that we can choose $M$ as large and then find constants $\mu_{0}>0, \varepsilon_{0}>0$ and $V_{0}>0$ (resp. $\kappa_{0}>0$ ), such that for all $\mu \geqq \mu_{0}$, $0<\varepsilon \leqq \varepsilon_{0}$ and $0<V \leqq V_{0}$ (resp. $0<\kappa \leqq \kappa_{0}$ ) we have

$$
T\left(S_{M}\right) \subset S_{M}
$$

It remains to show that $T\left(S_{M}\right)$ is precompact and that $T$ is continuous.

Again from the results in [4] and [5] we see that $\lambda(\Phi)$ and $\left|u_{\Phi}\right|_{C^{2}}$ are bounded by constants only depending on $M$, when $\Phi$ is in $S_{M}$. While the imbedding from $C^{2}(\bar{\Omega})$ in $C^{1, \alpha}(\bar{\Omega})$ is compact we conclude that $T\left(S_{M}\right)$ is precompact.

The continuity of $T$ follows from the uniqueness of the solution $u_{\Phi}$.

Thus $T$ has a fixed point $u=u_{\varepsilon}$, which is a solution to (1.2). Obviously, $u_{\varepsilon}$ has the right volume

$$
\int_{\Omega} u_{\varepsilon}-\psi d x=V
$$


and satisfies by (1.7)

$$
u_{\varepsilon}-\psi \geqq-3 \varepsilon \text {. }
$$

Letting now $\varepsilon$ tend to zero and $\mu$ tend to infinity, we get a Lipschitzsolution $u$ of the variational inequality (0.9), since the $C^{1}$ - norm of $u_{\varepsilon}$ is bounded independently of $\varepsilon$ and $\mu$. Furthermore, $u$ is also a solution to the variational inequality

$$
\langle A u+H(x, u)-\kappa u+\lambda, v-u\rangle \geqq 0 \quad \text { for all } v \in H^{1, \infty}(\Omega) \cap\{v \geqq \psi\}
$$

with some parameter $\lambda$.

The regularity of $u$ as stated in Theorem 0.1 now follows from the results in [13].

\section{A Priori Estimates for $|u|_{\Omega}$}

In order to get the estimate (1.7), we multiply (1.5) with

$$
w=\min (u-\psi+\delta, 0) \quad \delta \geqq \varepsilon .
$$

Introducing the notation $A(\delta)=\{x \in \Omega \mid u<\psi-\delta\}$ we then obtain by integration

$$
\begin{aligned}
\int_{A(\delta)} a^{i}(D u) \cdot D_{i}(u-\psi) d x & +\int_{\partial \Omega} \beta w d H_{n-1}+\int_{A(\delta)}(H(x, u)-\kappa \Phi+\lambda) w d x \\
& +\mu \cdot \int_{A(\delta)} \Theta_{\varepsilon}(u-\psi) \cdot(u-\psi+\delta) d x=0 .
\end{aligned}
$$

Now we observe that on $A(\delta)$ we have $\Theta_{\varepsilon}(u-\psi)=-1$ since $\delta \geqq \varepsilon$ and in addition $H(x, u) \leqq H(x, \psi)$ in view of the monotonicity of $H$. To estimate the boundary integral we use (0.6) and an inequality which is proved in ([6], Lemma 1):

$$
\int_{\partial \Omega} g d H_{n-1} \leqq \int_{\Omega}|D g| d x+c_{0} \int_{\Omega}|g| d x
$$

where $c_{0}=c_{0}(n, \partial \Omega)$.

We conclude for all $\delta \geqq \varepsilon$

$$
\begin{aligned}
a \cdot \int_{A(\delta)}|D u| d x & +\mu \cdot \int_{A(\delta)} \psi-u-\delta d x \leqq\left(1+2|D \psi|_{\Omega}\right)|A(\delta)| \\
& +\left(|H(\cdot, \psi(\cdot))|_{\Omega}+\kappa|\Phi|_{\Omega}+|\lambda|+c_{0}\right) \cdot \int_{A(\delta)} \psi-u-\delta d x
\end{aligned}
$$

where $|A(\delta)|$ denotes the Lebesgue measure in $\mathbb{R}^{n}$ of $A(\delta)$. Choosing now

$$
\mu \geqq \tilde{\mu}+|H(\cdot, \psi(\cdot))|_{\Omega}+\kappa|\Phi|_{\Omega}+|\lambda|+c_{0}
$$

we get with the triangle inequality

$$
\int_{\Omega}|D w| d x+\tilde{\mu} \cdot \int_{\Omega}|w| d x \leqq c\left(a, n,|D \psi|_{\Omega}\right) \cdot|A(\delta)|
$$


The Sobolev imbedding theorem yields

$$
\|w\|_{n / n-1}+\tilde{\mu} \cdot\|w\|_{1} \leqq c \cdot|A(\delta)| \quad \text { for all } \delta \geqq \varepsilon
$$

and by the Hölder inequality we obtain from (2.7)

and

$$
\left(\delta_{1}-\delta_{2}\right) \cdot\left|A\left(\delta_{1}\right)\right| \leqq c \cdot\left|A\left(\delta_{2}\right)\right|^{(n+1) / n}
$$

$$
\left(\delta_{1}-\delta_{2}\right) \cdot\left|A\left(\delta_{1}\right)\right| \leqq \tilde{\mu}^{-1} c \cdot \mid A\left(\delta_{2}\right) \quad \text { for all } \delta_{1} \geqq \delta_{2} \geqq \varepsilon \text {. }
$$

From a lemma due to Stampacchia $([17]$, Lemma 4.1) we now deduce

and

$$
u-\psi \geqq-2 \varepsilon-c \cdot|A(2 \varepsilon)|^{1 / n}
$$

$$
|A(2 \varepsilon)| \leqq e \cdot \exp \left(-\tilde{\mu} \cdot(e \cdot c)^{-1} \cdot \varepsilon\right) \cdot|A(\varepsilon)| \cdot
$$

Choosing now $\tilde{\mu}$ large enough, we conclude the inequality (1.7). Then $\mu$ depends on $|D \psi|_{\Omega}, a, n, \kappa|\Phi|_{\Omega},|H(\cdot, \psi)|_{\Omega}, \Omega, \lambda$ and $\varepsilon$. To establish the other bound (1.8) we multiply (1.5) with

$$
v=\max (u-\psi-k, 0)-\frac{1}{|\Omega|} \int_{\Omega} \max (u-\psi-k, 0) d x
$$

for any $k>0$. Observing $\int v d x=0$, we get by integration

$$
\begin{aligned}
\int_{A(k)} a^{i}(D u) \cdot D_{i}(u-\psi) d x+\int_{\partial \Omega} \beta v d H_{n-1} \\
\quad+\int_{\Omega}(H(x, u)-\kappa \Phi) \cdot v d x+\mu \cdot \int_{\Omega} \Theta_{\varepsilon}(u-\psi) \cdot v d x=0
\end{aligned}
$$

where now $A(k)$ denotes the subset of $\Omega$ where $u-\psi>k$.

Again using the inequalities (2.3) and (0.6) we can estimate the boundary integral by

$$
(1-a) \cdot \int_{A(k)}|D u| d x+|D \psi|_{\Omega} \cdot|A(k)|+2 c_{0} \cdot \int_{A(k)} \max (u-\psi-k, 0) d x
$$

The third term of (2.12) can be estimated as follows

$$
\begin{aligned}
\int_{\Omega} H(x, u) \cdot v d x= & \int_{A(k)} H(x, u) \cdot(u-\psi-k) d x \\
& -\int_{\Omega} \frac{1}{|\Omega|} \cdot \int_{A(k)} u-\psi-k d x H(\xi, u) d \xi \geqq \int_{A(k)} H(x, \psi) \cdot(u-\psi-k) d x \\
& -\int_{\{u>0\}} b(1+u) \frac{1}{|\Omega|} \cdot \int_{A(k)} u-\psi-k d x d \xi \\
& -\int_{\{u<0\}} H(\xi, 0) \frac{1}{|\Omega|} \cdot \int_{A(k)} u-\psi-k d x d \xi .
\end{aligned}
$$

This is a consequence of the assumptions $(0.7)$ and $(0.8)$. Finally we get 


$$
\begin{aligned}
& \left|\int_{\Omega} H(x, u) \cdot v d x\right| \\
& \quad \leqq\left(|H(\cdot, 0)|_{\Omega}+|H(\cdot, \psi)|_{\Omega}+b\left(1+\frac{1}{|\Omega|}\|u-\psi\|_{1}+|\psi|_{\Omega}\right)\right) \cdot \int_{A(k)} u-\psi-k d x .
\end{aligned}
$$

From the definition of $\Theta_{\varepsilon}$ in (1.3) we conclude that the last integral in (2.12) is positive. Thus, combining (2.12), (2.13) and (2.15) we derive

where

$$
a \cdot \int_{A(k)}|D u| d x \leqq 2 \cdot|D \psi|_{\Omega}|A(k)|+c_{1} \cdot \int_{A(k)} u-\psi-k d x
$$

$$
c_{1}=\left(2 \kappa|\Phi|_{\Omega}+2 c_{0}+|H(\cdot, 0)|_{\Omega}+|H(\cdot, \psi)|_{\Omega}+b\left(1+\frac{1}{|\Omega|}\|u-\psi\|_{1}+|\psi|_{\Omega}\right)\right) .
$$

Introducing the notation $w=\max (u-\psi-k, 0)$, the Hölder inequality yields

$$
a \cdot \int_{\Omega}|D w| d x \leqq c \cdot|A(k)|+c_{1}|A(k)|^{1 / n}\|w\|_{n / n-1}
$$

The Sobolev imbedding theorem leads to

$$
\|w\|_{n / n-1} \leqq c \cdot|A(k)|+c_{1} \cdot|A(k)|^{1 / n} \cdot\|w\|_{i / n-1}
$$

where now in $c_{1}$ is involved an additional factor depending on $a$ and $n$. To proceed further, we note that

and moreover

$$
|A(k)| \leqq \frac{1}{k} \cdot \int_{A(k)} u-\psi d x
$$

$$
\|u-\psi\|_{1} \leqq V+6 \varepsilon|\Omega|
$$

in view of the lower bound (1.7). Thus, if we choose

$$
k_{0}:=\left(2 c_{1}\right)^{n} \cdot(V+6 \varepsilon \cdot|\Omega|)
$$

we obtain from (2.19)

$$
\|w\|_{n / n-1} \leqq c \cdot|A(k)| \quad \text { for all } k \geqq k_{0} .
$$

Now we are in the same situation as in (2.7) and we conclude

$$
u-\psi \leqq k_{0}+c \cdot\left|A\left(k_{0}\right)\right|^{1 / n}
$$

where $c$ depends on $a, n$ and $|D \psi|_{\Omega}$.

Using again (2.20), we get by differentiating for $k_{0}$ the optimal estimate

$$
u-\psi \leqq \max \left(k_{0}, c\left(n, a,|D \psi|_{\Omega}\right) \cdot(V+6 \varepsilon|\Omega|)^{1 /(n+1)}\right)
$$

This completes the proof of Theorem 1.1. 


\section{A Priori Estimates for $|D u|_{\Omega}$}

We obtain a gradient bound for a solution $u$ of (1.4) by a modification of the methods in [4] and [18].

In view of the smoothness of $\partial \Omega$ we can extend $\beta$ and $\gamma$ into the whole domain $\Omega$ such that $\beta$ belonging to $C^{0,1}(\bar{Q})$ still satisfies $(0.6)$, and such that the vectorfield $\gamma$ is uniformly Lipschitz continuous in $\Omega$ and absolutely bounded by 1 . We shall use the following notations:

$S$ denotes the graph of $u$

$$
S=\left\{X=\left(x, x^{n+1}\right) \mid x \in \bar{\Omega}, x^{n+1}=u(x)\right\}
$$

and $\delta=\left(\delta_{1}, \ldots, \delta_{n+1}\right)$ the usual differential operators on $S$, i.e. for $g \in C^{1}\left(\bar{\Omega}^{n+1}\right)$ we have

$$
\delta_{i} g=D_{i} g-v_{i} \cdot \sum_{k=1}^{n+1} v^{k} \cdot D_{k} g
$$

where $v=\left(v_{1}, \ldots, v_{n+1}\right)$ is the exterior normal to $S$

$$
v=\left(1+|D u|^{2}\right)^{-1 / 2} \cdot\left(-D_{1} u, \ldots,-D_{n} u, 1\right)
$$

Following an idea of Ural'ceva [18], we want to prove that

$$
v:=\left(1+|D u|^{2}\right)^{1 / 2}+\beta \cdot D_{i} u \cdot \gamma^{i} \equiv W+\beta \cdot D_{i} u \cdot \gamma^{i}
$$

satisfies an estimate as stated in Theorem 1.2. This would be sufficient, since

Using the abbreviation

$$
|D u| \leqq W \leqq a^{-1} \cdot v \text {. }
$$

$$
\tilde{H}(x, u)=H(x, u)-\kappa \Phi+\lambda+\mu \cdot \Theta_{\varepsilon}(u-\psi)
$$

we state the following technical lemmata

Lemma 3.1. For any function $\mathrm{g} \in \mathrm{C}^{1}(\bar{\Omega})$ we have the Sobolev inequality

$$
\left(\int_{S}|g|^{n / n-1} d H_{n}\right)^{(n-1) / n} \leqq c_{2}(n) \cdot\left(\int_{S}|\delta g| d H_{n}+\int_{S}\left|\frac{\tilde{H}}{n}\right| g\left|d H_{n}+\int_{\partial \Omega}\right| g \mid \cdot W d H_{n-1}\right)
$$

where $H_{n}$ is the n-dimensional Hausdorff measure.

For functions vanishing on the boundary, this inequality was first established in [15], whereas a proof of the general case can be found in [4].

Lemma 3.2. For any positive function $\eta \in H^{1, \infty}(\Omega)$ we have the estimate

$$
\int_{\partial \Omega} v \cdot \eta d H_{n-1} \leqq \int_{S}|\delta \eta| d H_{n}+\int_{S}(|\tilde{H}|+|\delta \gamma|) \eta d H_{n} .
$$

A proof of this lemma can be found in [4].

Now we are going to estimate the function

$$
w=\log v
$$

Introducing the notations $z=\max (w-k, 0), A(k)=\{X \in S \mid w(x)>k\}$ and $|A(k)|$ $=H_{n}(A(k))$ we proceed exactly as in [13] in order to derive the inequality 


$$
\begin{aligned}
& \int_{A(k)}|\delta z|^{2} d H_{n}+\int_{A(k)}\left|\frac{\tilde{H}}{n}\right|^{2} \cdot z d H_{n} \\
& \quad \leqq c_{3} \cdot|A(k)|+\left(2 \kappa \cdot|D \Phi|_{\Omega}+c_{4}\right) \cdot \int_{A(k)} z d H_{n} \quad \text { for all } k \geqq k_{0}
\end{aligned}
$$

where

$$
\begin{aligned}
& c_{3}=c_{3}\left(\partial \Omega,|D \beta|_{\Omega},|\delta \gamma|_{\Omega}, n, a\right), \\
& c_{4}=c_{4}\left(|\delta \gamma|_{\Omega}, n, a,\left|\frac{\partial}{\partial x} H(\cdot, u)\right|_{\Omega},|D \beta|_{\Omega}\right), \\
& k_{0}=k_{0}\left(a, n,|D \psi|_{\Omega}\right) .
\end{aligned}
$$

To proceed further, we need the following lemma

Lemma 3.3. For arbitrary $\varepsilon>0$ the integral

can be estimated by

$$
\int_{A(k)} z d H_{n}=\int_{A(k)} w-k d H_{n}
$$

$$
\varepsilon \cdot \int_{A(k)}|\delta z|^{2} d H_{n}+\varepsilon \cdot \int_{A(k)}\left|\frac{\tilde{H}}{n}\right|^{2}(w-k) d H_{n}+c \cdot \varepsilon^{-1} \cdot|u-\psi|_{\Omega}^{2} \cdot|A(k)|
$$

provided $k \geqq k_{0}=k_{0}\left(a, c_{0},|D \psi|_{\Omega}\right)$.

Proof of Lemma 3.3. We consider the identity

$$
\begin{aligned}
& \int_{\Omega} a^{i} D_{i} \eta d x+\int_{\Omega} H(x, u) \eta-\kappa \Phi \cdot \eta+\lambda \eta d x \\
& \quad+\mu \cdot \int_{\Omega} \Theta_{\varepsilon}(u-\psi) \eta d x+\int_{\partial \Omega} \beta \eta d H_{n-1}=0 \quad \text { for all } \eta \in H^{1, \infty}(\Omega) .
\end{aligned}
$$

Using this identity with $\eta=(u-\psi) \cdot z$, we obtain with the help of (2.3)

$$
\begin{aligned}
\int_{\{w>k\}}|D u|^{2} \cdot W^{-1} z d x & \leqq \int_{\{w>k\}} a^{i} D_{i} \psi \cdot z d x-\int_{\{w>k\}} a^{i}(u-\psi) D_{i} w d x \\
& +\int_{\{w>k\}}|\tilde{H}||u-\psi| z d x+(1-a)\left(\int_{\{w>k\}}|D u| z d x+\int_{\{w>k\}}|D \psi| z d x\right. \\
& \left.+\int_{\{w>k\}}|\mathcal{u}-\psi||D w| d x+c_{0} \cdot \int_{\{w>k\}}|u-\psi| z d x\right) .
\end{aligned}
$$

Thus, we conclude

$$
\begin{aligned}
& a \cdot \int_{\{w>k\}} W z d x \leqq \int_{\{w>k\}} z d x+2|D \psi|_{\Omega} \cdot \int_{\{w>k\}} z d x \\
& +\varepsilon \cdot \int_{\{w>k\}}\left|\frac{\tilde{H}}{n}\right|^{2} z d x+(4 \varepsilon)^{-1} \cdot|u-\psi|_{\Omega}^{2} \cdot \int_{\{w>k\}} z d x \\
& +\varepsilon \cdot \int_{\{w>k\}}|\delta w|^{2} W d x+(4 \varepsilon)^{-1} \cdot|u-\psi|_{\Omega}^{2} \cdot \int_{\{w>k\}} W d x \\
& +c_{0} \cdot|u-\psi|_{\Omega}^{2} \cdot \int_{\{w>k\}} W d x+c_{0} \cdot \int_{\{w>k\}} z^{2} W^{-1} d x
\end{aligned}
$$


and since $z \leqq W$ for $k \geqq k_{0}\left(a, c_{0},|D \psi|_{\Omega}\right)$ we obtain

$$
\int_{\{w>k\}} W z d x \leqq \varepsilon \cdot \int_{\{w>k\}}|\delta w|^{2} W d x+\varepsilon \cdot \int_{\{w>k\}}\left|\frac{\tilde{H}}{n}\right|^{2} \cdot z d x+c \cdot \varepsilon^{-1} \cdot|u-\psi|_{\Omega}^{2} \cdot|A(k)|
$$

from which the assertion follows.

Applying now this lemma to (3.10) we derive

$$
\int_{A(k)}|\delta z|^{2} d x+\int_{A(k)}\left|\frac{\tilde{H}}{n}\right|^{2} \cdot z d x \leqq\left(c+\kappa|D \Phi|_{\Omega} \cdot|u-\psi|_{\Omega}\right)^{2} \cdot|A(k)| .
$$

Moreover, from the Sobolev imbedding, Lemma 3.1 and from Lemma 3.2 we conclude

$$
\begin{aligned}
& \left(\int_{S}|z|^{n / n-1} d H_{n}\right)^{(n-1) / n} \\
& \quad \leqq c(n) \cdot\left(\int_{S}|\delta z| d H_{n}+\int_{S}\left|\frac{\tilde{H}}{n}\right| z d H_{n}+\int_{\partial \Omega} W z d H_{n-1}\right) \\
& \quad \leqq c\left(\left(\int_{S}|\delta z|^{2} d H_{n}\right)^{1 / 2} \cdot|A(k)|^{1 / 2}+\varepsilon \cdot \int_{S}\left|\frac{\tilde{H}}{n}\right|^{2} \cdot z d H_{n}+c_{\varepsilon} \cdot \int_{S} z d H_{n}\right) .
\end{aligned}
$$

To estimate the first integral on the right side of (3.18), we remark that from (3.17) we have

$$
\left(\int_{S}|\delta z|^{2} d H_{n}\right)^{1 / 2} \leqq\left(c+\kappa|D \Phi|_{\Omega} \cdot|u-\psi|_{\Omega}\right) \cdot|A(k)|^{1 / 2} .
$$

Thus we obtain from (3.17) and (3.18)

$$
\begin{aligned}
& \left(\int_{S}|z|^{n / n-1} d H_{n}\right)^{(n-1) / n}+\int_{S}|\delta z|^{2} d H_{n}+\int_{S}\left|\frac{\tilde{H}}{n}\right|^{2} \cdot z d H_{n} \\
& \leqq\left(c+\kappa|D \Phi|_{\Omega} \cdot|u-\psi|_{\Omega}\right)|A(k)|+\varepsilon \cdot \int_{S}\left|\frac{\tilde{H}}{n}\right|^{2} \cdot z d H_{n}+c_{\varepsilon} \cdot \int_{S} z d H_{n} .
\end{aligned}
$$

Then, again using Lemma 3.3 we obtain

$$
\left(\int_{S}|z|^{n / n-1} d H_{n}\right)^{(n-1) / n} \leqq\left(c+\kappa|D \Phi|_{\Omega} \cdot|u-\psi|_{\Omega}\right) \cdot|A(k)|
$$

and from the Hölder inequality we get

$$
\int_{S} z d H_{n} \leqq\left(c+\kappa|D \Phi|_{\Omega} \cdot|u-\psi|_{\Omega}\right) \cdot|A(k)|^{1+1 / n} \quad \text { for all } k \geqq k_{0} .
$$

By another use of Stampacchia's lemma we conclude

$$
w=\log v \leqq k_{0}+\left(c+\kappa|D \Phi|_{\Omega} \cdot|u-\psi|_{\Omega}\right) \cdot|S|^{1 / n}
$$

where

$$
\begin{aligned}
k_{0} & =k_{0}\left(a, n,|D \psi|_{\Omega}, \partial \Omega\right) \\
c & =c\left(\left|\frac{\partial}{\partial x} H(\cdot, u)\right|_{\Omega}, a, n,|\delta \gamma|_{\Omega},|D \beta|_{\Omega}, \Omega\right) .
\end{aligned}
$$


It remains to establish a bound for $|S|=\int_{\Omega} W d x$.

To accomplish this, we use the identity (3.13) with

We get

$$
\eta=u-\psi-\frac{V}{|\Omega|}
$$

$$
\begin{aligned}
\int_{\Omega}|D u|^{2} W^{-1} d x \leqq & \int_{\Omega} a^{i} D_{i} \psi d x+\int_{\Omega}|H|\left|u-\psi-\frac{V}{|\Omega|}\right| d x \\
& +\kappa|\Phi|_{\Omega} \cdot \int_{\Omega}\left|u-\psi-\frac{V}{|\Omega|}\right| d x-\lambda \cdot \int_{\Omega} u-\psi-\frac{V}{|\Omega|} d x \\
& -\mu \cdot \int_{\Omega} \Theta_{\varepsilon}(u-\psi)\left(u-\psi-\frac{V}{|\Omega|}\right) d x+\int_{\Omega}|D \psi| d x \\
& +c_{0} \cdot \int_{\Omega}\left|u-\psi-\frac{V}{|\Omega|}\right| d x+(1-a) \cdot \int_{\Omega}|D u| d x .
\end{aligned}
$$

By the definition of $\Theta_{\varepsilon}$ and $V$ the terms with $\lambda$ and $\mu$ may be neglected. Thus, we have

$$
\int_{\Omega} W d x \leqq c\left(|D \psi|_{\Omega},|\Omega|, a, n, c_{0},|H|, N\right)
$$

where $N$ is an arbitrary upper bound for $V$ and $|u-\psi|_{\Omega}$. This proves Theorem 1.2.

\section{Solutions to the Equation}

Let $\Omega \subset \mathbb{R}^{n}, n \geqq 2$ be of class $C^{2, \alpha}$ and assume that $\beta$ and $H$ satisfy the conditions $(0.5)-(0.8)$. Then we consider the boundary value problems

We shall prove:

$$
\begin{aligned}
A u_{\kappa}+H\left(x, u_{\kappa}\right)-\kappa u_{\kappa}+\lambda_{\kappa}=0 & \text { in } \quad \Omega \\
-a^{i}\left(D u_{\kappa}\right) \cdot \gamma_{i}=\beta & \text { on } \quad \partial \Omega
\end{aligned}
$$

Theorem 4.1. Assume there is a solution $u_{0} \in C^{2, \alpha}(\bar{\Omega})$ to the problem $(4.1 ; 0)$. Then we can find $\kappa_{0}>0$ so that for all $0 \leqq \kappa \leqq \kappa_{0}$ there is some $\lambda_{\kappa}$ and a function $u_{k} \in C^{2, \alpha}(\bar{\Omega})$ satisfying $(4.1 ; \kappa)$.

We shall discuss the existence of a solution $u_{0}$ in the case $\kappa=0$ at the end of this section.

Proof of Theorem 4.1. Let $\Omega$ be connected. In the other case we can carry out the proof in every component.

We consider the variational inequality

$$
\left\langle A u_{\kappa}+H\left(x, u_{\kappa}\right)-\kappa u_{\kappa}, v-u_{\kappa}\right\rangle \geqq 0 \quad \text { for all } v \in K
$$

where

$$
K:=H^{1, \infty}(\Omega) \cap\{v \geqq \psi\} \cap\left\{\int_{\Omega} v-\psi d x=v\right\}
$$


We take $\psi \equiv u_{0}-1$ and $V=|\Omega|$, so that $u_{0}$ is a solution to $(4.2 ; 0)$.

By Theorem 0.1 , the problem $(4.2 ; \kappa)$ admits a solution $u_{\kappa} \in H^{2, \infty}(\Omega)$ if $\kappa$ is small enough, say $0 \leqq \kappa \leqq \kappa_{1}$. Moreover, there exists a Lagrange parameter $\lambda_{k}$ such that $u_{\kappa}$ solves

$$
\left\langle A u_{\kappa}+H\left(x, u_{\kappa}\right)-\kappa u_{\kappa}+\lambda_{\kappa}, v-u_{\kappa}\right\rangle \geqq 0 \quad \text { for all } v \in H^{1, \infty}(\Omega) \cap\{v \geqq \psi\}
$$

and we have

$$
\left|u_{\kappa}\right|_{C^{1}} \leqq M \quad \text { for all } 0 \leqq \kappa \leqq \kappa_{1}
$$

for some constant $M$ (see Sect. 2,3).

It is our claim to show that for $\kappa$ small enough the functions $u_{\kappa}$ lie strictly above the obstacle $\psi \equiv u_{0}-1$ and therefore solve the Eq. $(4.1 ; \kappa)$. To accomplish this, we need

Lemma 4.1. For $\kappa$ small enough, a solution to $(4.2 ; \kappa)$ is unique in the class of functions satisfying (4.5).

Proof of Lemma 4.1. Let $\tilde{u}_{\kappa}$ be another solution to $(4.2 ; \kappa)$, satisfying (4.5). We obtain

$$
\int_{\Omega}\left(a^{i}\left(D u_{\kappa}\right)-a^{i}\left(D \tilde{u}_{\kappa}\right)\right)\left(D_{i} u_{\kappa}-D_{i} \tilde{u}_{\kappa}\right) d x \leqq \kappa \cdot \int_{\Omega}\left|u_{\kappa}-\tilde{u}_{\kappa}\right|^{2} d x .
$$

In view of (4.5) the first term can be estimated from below by

$$
c(M) \cdot \int_{\Omega}\left|D\left(u_{\kappa}-\tilde{u}_{\kappa}\right)\right|^{2} d x
$$

and the Poincare inequality shows that for small $\kappa_{1}$ we have $u_{\kappa}-\tilde{u}_{\kappa}=$ const. The assertion now follows from the fact that $u_{\kappa}$ and $\tilde{u}_{\kappa}$ have the same volume.

From this Lemma 4.1 we conclude immidiately that the map

$$
\begin{aligned}
\kappa & \rightarrow u_{\kappa} \\
{\left[0, \kappa_{1}\right] } & \rightarrow C^{0}(\bar{\Omega})
\end{aligned}
$$

is continuous and therefore $u_{\kappa}$ tends to $u_{0}$ uniformly when $\kappa$ goes to zero. Thus, there is some $\kappa_{0} \leqq \kappa_{1}$ such that for all $\kappa \leqq \kappa_{0}$ the function $u_{\kappa}$ lies strictly above the obstacle $u_{0}-1$ and is then a global regular solution to $(4.1 ; \kappa)$.

Remark. If $H(x, t)=H(x)$, we can always choose $\lambda_{\kappa}=0$ in $(4.1 ; \kappa)$. We have only to add a suitable constant to $u_{\kappa}$.

Now we want to discuss the case $\kappa=0$.

Giusti [9] considered the functional

$$
F(v)=\int_{\Omega}\left(1+|D v|^{2}\right)^{1 / 2} d x+\iint_{\Omega}^{v} H(x, t) d t d x+\int_{\partial \Omega} \beta v d H_{n-1}
$$

under the following assumption:

There exist two positive constants $\varepsilon_{0}$ and $t_{0}$ such that for every Cacciopoli set $B \subset \Omega$ we have 


$$
\begin{gathered}
\int_{B} H\left(x, t_{0}\right) d x+\int_{\partial \Omega} \beta \chi_{B} d H_{n-1} \leqq-\left(1-\varepsilon_{0}\right) \int_{\Omega}\left|D \chi_{B}\right| d x \\
\int_{B} H\left(x,-t_{0}\right) d x+\int_{\partial \Omega} \beta \chi_{B} d H_{n-1} \leqq\left(1-\varepsilon_{0}\right) \int_{\Omega}\left|D \chi_{B}\right| d x .
\end{gathered}
$$

It was shown that $F$ has a minimum $u$ in $B V(\Omega)$ which is bounded by a constant depending only on $\varepsilon_{0}, t_{0}$ and $\|u\|_{1}$. Moreover, $u$ is of class $C^{2, \alpha}$ in the interior of $\Omega$.

Theorem 4.2. The minimum $u$ of $F$ is in $C^{2, \alpha}(\bar{\Omega})$ and satisfies

$$
\begin{aligned}
A u+H(x, u)=0 & \text { in } \Omega \\
-a^{i}(D u) \cdot \gamma_{i}=\beta & \text { on } \partial \Omega .
\end{aligned}
$$

Proof. of Theorem 4.2. In view of Giusti [9] we have

$$
\sup _{\Omega}|u| \leqq c\left(\varepsilon_{0}, t_{0},\|\mathcal{u}\|_{1}\right)=: M
$$

Now let $\eta$ and $\zeta$ be smooth monotone functions on $\mathbb{R}$ with

Then, the function

$$
\begin{gathered}
\zeta(t)=\left\{\begin{array}{cc}
t & |t| \leqq M+1 \\
M+2 & t \geqq M+3 \\
-M-2 & t \leqq-M-3,
\end{array}\right. \\
\eta(t)=\left\{\begin{array}{cc}
0 & |t| \leqq M+1 \\
t-(M+2) & t \geqq M+3 \\
t+(M+2) & t \leqq-M-3 .
\end{array}\right.
\end{gathered}
$$

satisfies

$$
\hat{H}(x, t)=H(x, \zeta(t))+\eta(t)
$$

and

$$
\hat{H}(x, u)=H(x, u)
$$

$$
\frac{\partial \hat{H}}{\partial t} \geqq 0, \quad \frac{\partial \hat{H}}{\partial t}=1 \quad \text { for } \quad|t| \geqq M+3 .
$$

We consider the new problem

$$
\begin{aligned}
A v+\hat{H}(x, v)+\delta v & =0 & & \text { in } \Omega \\
-a^{i} \cdot \gamma_{i} & =\beta & & \text { on } \partial \Omega
\end{aligned}
$$

where the term $\delta v$ has only to ensure the uniqueness of a solution. In view of the properties of $\hat{H}$ in (4.17) we have global a priori estimates for $|v|_{\Omega}$ and $|D v|_{\Omega}$ independently of $\delta$. (For a proof of the gradient bound see Sect. 3 or [4].)

By a result due to Gerhardt [4] there is a solution $u_{\delta} \in C^{2, \alpha}(\bar{\Omega})$ of $(4.18)$ for any $\delta>0$. The $u_{\delta}$ are uniformly bounded in $C^{2, \alpha}(\bar{\Omega})$ and thus the boundary value problem

$$
\begin{aligned}
A v+\hat{H}(x, v)=0 & \text { in } \Omega \\
-a^{i} \cdot \gamma_{i}=\beta & \text { on } \partial \Omega
\end{aligned}
$$


admits a solution $\hat{u} \in C^{2, \alpha}(\bar{\Omega})$. But in view of (4.16) we have $u-\hat{u}=$ const and hence $u \in C^{2, \alpha}(\bar{\Omega})$.

Now the question arises whether there are simple cases, where the condition (4.10) can be verified. If $\beta$ and $H$ are constants, we have the problem

where

$$
\begin{gathered}
A u+\lambda=0 \quad \text { in } \Omega \\
-a^{i} \gamma_{i}=\beta_{0} \quad \text { on } \partial \Omega
\end{gathered}
$$

$$
\lambda=-\frac{|\partial \Omega|}{|\Omega|} \cdot \beta_{0}, \quad \beta_{0}<1
$$

In [10] Giusti and Weinberger considered 'maximal domains', i.e. the case $\beta_{0}$ $=1$. In particular they showed in the case $n=2$ that for all $\beta_{0}$ there is a solution of (4.20), if $\Omega$ is convex and the curvature of $\partial \Omega$ is always less than $|\partial \Omega| /|\Omega|$.

Chen showed in [1], that whenever a disk of radius $|\Omega||\partial \Omega|$ can be rolled around $\partial \Omega$ in the interior of $\Omega$, then the condition (4.10) is satisfied and therefore a solution of (4.20) exists.

Finally, Finn [3] established general geometric criteria that suffice for the existence of a solution to (4.20), corresponding to any $\beta_{0}$.

\section{A Result in the Dirichlet Case}

Let $\varphi$ be a function in $C^{2, \alpha}(\bar{\Omega})$ and assume that the following additional conditions are valid:

$$
\begin{aligned}
& \int_{\Omega} H(x, 0) v d x \leqq\left(1-\varepsilon_{0}\right) \cdot \int_{\Omega}|D v| d x, \quad \varepsilon_{0}>0 \\
& (n-1) \cdot K(x)>|H(x, \varphi(x))| \text { for all } x \in \partial \Omega
\end{aligned}
$$

where $K(x)$ is the mean curvature of $\partial \Omega$ in $x$.

Theorem 5.1. There exists some $\kappa_{0}>0$ so that for every $0 \leqq \kappa \leqq \kappa_{0}$ the problem

has a solution $u_{\kappa} \in C^{2, \alpha}(\bar{\Omega})$.

$$
\begin{aligned}
A u_{\kappa}+H\left(x, u_{\kappa}\right)-\kappa u_{\kappa}=0 & \text { in } \Omega \\
u_{\kappa}=\varphi & \text { on } \partial \Omega
\end{aligned}
$$

As in Sect. 1 the proof depends on suitable a priori estimates for the $C^{1}$ norm of solutions to the related problem

$$
\begin{aligned}
A u_{\Phi}+H\left(x, u_{\Phi}\right)-\kappa \Phi & =0 & & \text { in } \Omega \\
u_{\Phi} & =\varphi & & \text { on } \partial \Omega
\end{aligned}
$$

where $\Phi$ is some function in $C^{1, \alpha}(\bar{\Omega})$.

It is well known, that in view of (5.1) for every $\Phi$ the $\kappa$ can be taken as small that (5.4) has a unique global regular solution $u_{\Phi}$.

Now let

$$
\Phi \in S_{M}:=\left\{\left.v \in C^{1, \alpha}(\bar{\Omega})|| v\right|_{C^{1}} \leqq M\right\}
$$


and

$$
0 \leqq \kappa \leqq \kappa_{1}=\kappa_{1}(M)
$$

where $\kappa_{1}(M)$ is as small that we can define an operator

$$
\begin{aligned}
T: S_{M} & \rightarrow C^{1, \alpha}(\bar{\Omega}) \\
\Phi & \rightarrow u_{\Phi} .
\end{aligned}
$$

If we can show that for some $M$ large enough and $\kappa_{1}(M)$ small enough we have

$$
T\left(S_{M}\right) \subset S_{M}
$$

then we derive from Lemma 1.2, that $T$ has a fixed point in $S_{M}$, which is of course a regular solution to (5.3). The inclusion (5.8) may be derived from the following a priori estimates:

Lemma 5.1. Let $u_{\Phi}$ be a solution to (5.4). We then have for small $\kappa$ :

$$
|u|_{\Omega} \leqq \max _{\partial \Omega} \varphi+\left(c(n) \cdot \varepsilon_{0}-\kappa|\Phi|_{\Omega}|\Omega|^{1 / n}\right)^{-1}|\Omega|^{1 / n} .
$$

Proof of Lemma 5.1. We multiply (5.4) with $w=\max \left(u_{\Phi}-k, 0\right)$ for $k \geqq k_{0}=\max _{\partial \Omega} \varphi$ and denote by $A(k)$ the set $\{x \in \Omega \mid u>k\}$. We get

$$
\int_{A(k)} a^{i}\left(D u_{\Phi}\right) \cdot D_{i} u_{\Phi} d x+\int_{A(k)} H\left(x, u_{\Phi}\right) \cdot\left(u_{\Phi}-k\right) d x=\kappa \cdot \int_{\Omega} \Phi w d x
$$

from which we derive in view of $(0.7)$ and (5.1)

$$
c(n) \cdot \varepsilon_{0} \cdot\|w\|_{n /(n-1)} \leqq \int_{A(k)}\left|D u_{\Phi}\right| d x \leqq|A(k)|+\kappa|\Phi|_{\Omega}\|w\|_{n /(n-1)}|A(k)|^{1 / n}
$$

Since $\kappa$ is small, we obtain

$$
(h-k)|A(h)| \leqq\left(c(n) \cdot \varepsilon_{0}-\kappa|\Phi|_{\Omega}|\Omega|^{1 / n}\right)^{-1}|A(k)|^{1+1 / n} .
$$

The upper bound now follows as in previous sections with the help of Stampacchia's result and the lower bound can be derived by similar calculations.

A gradient bound follows from results due to Serrin [16] and Giaquinta [7]. We derive

Lemma 5.2. We can take $M$ as large and $\kappa_{0}(M)$ as small that

$$
\left|D u_{\Phi}\right|_{\Omega} \leqq M
$$

holds for all $\Phi \in S_{M}$ and all $0 \leqq \kappa \leqq \kappa_{0}$.

Proof of Lemma 5.2. In the interior of $\Omega$ the result follows from a local version of the gradient estimate in Sect. 3. At the boundary $\partial \Omega$ the gradient estimate depends on the existence of suitable barrier functions. It was shown in [7] and [16], that such barriers always exist, provided the Serrin condition is satisfied. In our case this condition takes the form

$$
(n-1) \cdot K(x) \geqq|H(x, \varphi)-\kappa \Phi| \quad \text { for all } x \in \partial \Omega .
$$


Thus, as a first step we have to choose $\kappa_{0}$ and $M$ such that

$$
\kappa_{0} \cdot M \leqq \min _{\partial \Omega}((n-1) \cdot K(x)-H(x, \varphi(x))) .
$$

From this we obtain a bound $N$ for $\left|D u_{\Phi}\right|_{\Omega}$, depending on known quantities and on the product $\left(\kappa_{0} \cdot M\right)$ (see [7]). Then we may choose $M>N$. We have only to ensure, that the product $\left(\kappa_{0} \cdot M\right)$ does not enlarge. But this can be managed by a suitable choice of $\kappa_{0}$.

This completes the proof of Theorem 5.1.

\section{References}

1. Chen, J.: On the existence of capillary surfaces in the absence of gravity. Pacific J. Math. 88, 323-361 (1980)

2. Concus, P., Finn, R.: On capillary free surfaces in a gravitational field. Acta Math. 132, 207$223(1974)$

3. Finn, R.: A subsidiary variational problem and existence criteria for capillary surfaces. Preprint

4. Gerhardt, C.: Global regularity of the solutions to the capillarity problem. Ann. Scuola Norm. Sup. Pisa Ser. (4) 3, 157-175 (1976)

5. Gerhardt, C.: On the capillarity problem with constant volume. Ann. Scuola Norm. Sup. Pisa Ser. (4) 2, 304-320 (1975)

6. Gerhardt, C.: Existence and regularity of capillary surfaces. Boll. Un. Mat. Ital. 10, 317-335 (1974)

7. Giaquinta, M.: On the Dirichlet problem for surfaces of prescribed mean curvature, Manuscripta Math. 12, 73-86 (1974)

8. Gilbarg, D., Trudinger, N.S.: Elliptic partial differential equations of second order. Grundl. Math. Wiss. 224. Berlin-Heidelberg-New York: Springer 1977

9. Giusti, E.: Boundary value problems for non-parametric surfaces of prescribed mean curvature. Ann. Scuola Norm. Sup. Pisa Ser. (4) 3, 501-548 (1976)

10. Giusti, E.: On the equation of surfaces of prescribed mean curvature. Invent. Math. 46, 111-137 (1978)

11. Giusti, E.: The pendent water drop. A direct approach. Boll. Un. Mat. Ital. 17, 458-465 (1980)

12. Gonzales, E.: Sul problema della goccia appoggiata. Rend. Sem. Mat. Univ. Padova 55, 289302 (1976)

13. Huisken, G.: Capillary surfaces over obstacles. To appear in Pacific J. Math,

14. Massari, U., Pepe, L.: Su di una formulazione variazionale del problema dei capillari in assenza di gravitá. Ann. Univ. Ferrara Sez. VIII 20, 33-42 (1974)

15. Michael, J.H., Simon, L.M.: Sobolev and mean value inequalities on generalized submanifolds of $\mathbb{R}^{n}$. Comm. Pure Appl. Math. 26, 361-379 (1973)

16. Serrin, J.: The problem of Dirichlet for quasilinear elliptic differential equations with many independent variables. Philos. Trans. Roy. Soc. London Ser. A 264, 313-496 (1969)

17. Stampacchia, G.: Equations elliptiques du second ordre à coefficients discontinus. Montréal: Les Presses de l'Université 1966

18. Ural'ceva, N.N.: The solvability of the capillarity problem. Vestnik Leningrad Univ. Mat. Meh. Astronom. 4, 54-64 (1973) [Russian] 